\title{
Anaplastic transition within the cancer microenvironment in early-stage oral tongue squamous cell carcinoma is associated with local recurrence
}

\author{
KOHEI OKUYAMA ${ }^{1}$, KEIJI SUZUKI ${ }^{2}$, SOUICHI YANAMOTO ${ }^{1}$, TOMOFUMI NARUSE ${ }^{1}$, \\ HIROKI TSUCHIHASHI $^{1}$, SHUNICHI YAMASHITA ${ }^{2}$ and MASAHIRO UMEDA ${ }^{1}$ \\ ${ }^{1}$ Department of Clinical Oral Oncology, Nagasaki University Graduate School of Biomedical Sciences; \\ ${ }^{2}$ Department of Radiation Medical Sciences, Atomic Bomb Disease Institute, \\ Nagasaki University, Nagasaki-shi, Nagasaki 852-8588, Japan
}

Received May 24, 2018; Accepted June 29, 2018

DOI: 10.3892/ijo.2018.4515

\begin{abstract}
The cancer microenvironment (CME) promotes malignant progression of cancer cells by stimulating cell growth, migration and invasion. Cancer-associated fibroblasts (CAFs), prominent features of the CME, interact directly with cancer cells and facilitate epithelial-mesenchymal transition (EMT). The present study examined the spatial distribution of CAFs and EMT on cancer cells in patients with early-stage tongue squamous cell carcinoma (TSCC) and their association with local recurrence. The present study included 14 patients with early-stage TSCC who had undergone glossectomy between 2006 and 2015, of which 7 experienced local recurrence (LR group) and $7 \mathrm{did}$ not (control group). Multiple immunofluorescent analysis (MIA) of PCNA, aSMA, vimentin, E-cadherin and cytokeratin 14 (CK14) was performed on slides obtained from surgical specimens to identify the expression of various cell-specific markers. The number of CAFs in the CME was significantly increased in the LR group $(\mathrm{P}=0.001)$. Furthermore, the neighbouring cancer cells were positive for vimentin expression, indicating EMT. However, the present study also identified concurrent expression of CK14 in all vimentin-positive cancer cells, whilst epithelial markers, including E-cadherin, were expressed in certain vimentin-positive cancer cells. Concurrent expression of CK14 and vimentin is not defined as EMT or partial EMT. Therefore, the present study proposed a novel mechanism of anaplastic transition (APT), in which epithelial cancer cells
\end{abstract}

Correspondence to: Professor Kohei Okuyama, Department of Clinical Oral Oncology, Nagasaki University Graduate School of Biomedical Sciences, Nagasaki University, 1-7-1 Sakamoto, Nagasaki-shi, Nagasaki 852-8588, Japan

E-mail: okuyamak@nagasaki-u.ac.jp

Key words: anaplastic transition, tongue squamous cell carcinoma, local recurrence, epithelial-mesenchymal transition, cancerassociated fibroblasts concurrently develop mesenchymal features, which is achieved by pathways other than EMT. APT is characterized such that epithelial cancer cells differentiate into more primitive states, which is different from EMT or partial EMT, and it may be associated with LR. The concept aids in improving knowledge regarding tumor recurrence in patients with early-stage TSCC.

\section{Introduction}

Tongue squamous cell carcinoma (TSCC) is the most prevalent type of oral malignant tumor. Despite advances in recent decades in diagnosis, as a result of improved imaging modalities and surgical techniques, the survival of TSCC patients has remained unchanged (1-3). This is mainly due to the high recurrence rate and the high risk of cervical lymph node metastasis or distant metastasis (1-3).

There is substantial evidence indicating that interactions between cancer cells and the cancer microenvironment (CME) are of great importance to the development and progression of cancer. Cancer cells alter the surrounding cancer stroma. Cancer stromal cells and cytokines, in turn, promote cancer progression and the acquisition of invasive properties $(4,5)$. Recently, it has become apparent that carcinomas recruit benign microenvironment-supporting cells to facilitate invasion and metastasis $(6,7)$. Cancer-associated fibroblasts (CAFs) are tumor-associated fibroblasts with myofibroblast-like phenotypes that can be observed within the CME, particularly close to the cancer nests (7-9). It has been reported that CAFs are derived from normal cancer stromal fibroblasts under the direct impact of cancer cell-derived cytokines, and they further facilitate local and distant migration, as well as aiding in the suppression of the host immune response (9-13). CAFs exhibit changes in protein expression levels that represent an activated myofibroblastic phenotype, which typically involves the upregulation of $\alpha$-smooth muscle actin ( $\alpha$ SMA) $(14,15)$. They also promote cancer progression via the stimulation of epithelial cell growth, migration and invasion by imitating activated wound repair fibroblasts (5,16-18). Experiments in vitro have also revealed that CAFs can stimulate the proliferation and invasion of TSCC cells $(19,20)$. 
The most striking role of CAFs in the CME is the stimulation of the epithelial-mesenchymal transition (EMT), which is a necessary step toward invasion, metastasis and apoptosis resistance $(7,8,9,21)$. Although the definition of EMT is broad, it is generally agreed that it involves loss of E-cadherin expression resulting in loss of cell-cell contact between cancer epithelial cells, cytoskeleton reorganization via switching from cytokeratin $(\mathrm{CK})$ to vimentin intermediate filaments, loss of apical-basal polarity, acquisition of a fibroblast-like cell shape and increased cellular motility $(22,23)$. In the EMT process, cancer cells with decreased E-cadherin expression are primarily located at the cancer periphery and directly contact with CAFs, indicating that EMT may be modulated by CAFs (8). To achieve EMT, the coordinated expression of several sets of genes and signalling pathways is required, a number of which have been demonstrated to regulate specific aspects of malignant transformation and cancer progression $(24,25)$. One pathway involves the activation of transcription factors, including Snail, Slug and Twist, which cause transcriptional repression of E-cadherin, a defining step in $\operatorname{EMT}(22,23,26)$.

In our clinical experience, there have been several cases of early-stage TSCC with local recurrence in the early postoperative period, despite tumor resection with adequate safety and negative margins. We hypothesised that such aggressive tumors may be accompanied by the process of EMT stimulation within the CME; therefore, evaluation of the CME may provide clues that enable the detection of prognostic factors for the local recurrence of TSCC. The present study used multiple immunofluorescent analysis (MIA), which enables detailed and concurrent examination of multiple markers, to evaluate the distribution of CAFs in the CME using tissue specimens obtained from the early-stage TSCC patients with local recurrence who underwent glossectomy with clear margins. The results demonstrated that localised distribution of CAFs in CME was significantly different between cases with local recurrence and those without. It was demonstrated that the cancer cells neighbouring CAFs exhibited simultaneous expression of E-cadherin and vimentin, in keeping with a study by Wang et al (27); however, all vimentin-positive cancer cells continued to express the epithelial marker, CK, and even certain vimentin-positive cells continued to express E-cadherin. To date, the expression of vimentin and the loss of E-cadherin in epithelial cancer cells have been used to diagnose EMT, direct conversion of epithelial cells to mesenchymal cells. However, such a diagnosis does not take into account the possibility that epithelial cancer cells are able to transform back into epiblasts, which are undifferentiated CK-positive cells expressing vimentin.

In the present study, we propose a novel concept referred to as anaplastic transition (APT), in which epithelial cancer cells dedifferentiate into primitive states. This proposal was based on the observation that the loss of E-cadherin expression does not necessarily indicate a loss of $\mathrm{CK}$, and that these cells retain epithelial and mesenchymal CKs.

\section{Materials and methods}

Patients and samples. A total of 10 male and 4 female patients with early-stage TSCC were included in the present study. The mean age of the patients at the time of surgery was 61.4 years (range, 39-79 years) in the LR group and 64.1 years (range, 52-86 years) in the control group. All samples in the present study were obtained from 14 patients who were clinically diagnosed with early-stage (cT1-2) squamous cell carcinoma and who underwent glossectomy with adequate safety and negative margins without preoperative neoadjuvant chemotherapy and/or radiation at the department of Clinical Oral Oncology in Nagasaki University Hospital between April 2006 and December 2015. Tumors in all patients were classified according to the World Health Organization (WHO) classification by a pathologist who was blinded to the clinical findings. The pathological Tumor-Node-Metastasis (TNM) classification was established using the International System for Staging adopted by the American Joint Committee on Cancer and the Union for International Cancer Control (8th edition) $(28,29)$. This retrospective study includes 7 cases of TSCC who experienced local recurrence (LR group) following appropriate resection with clear safety margins $(>10 \mathrm{~mm}$ ) and 7 randomly selected cases of TSCC without local recurrence who underwent glossectomy over the same period (control group). Sex, age, TNM classification, clinical growth pattern, differentiation, vascular/lymphatic perineural invasion, and outcomes were evaluated.

Postoperative follow-up was performed using contrastenhanced computed tomography (CT) and examination by ultrasound of the neck for at least 3 months. LR was defined as the detection of a mass on CT images and/or clinical inspection.

Ethical approval. The present study was approved by the Institutional Review Board of Nagasaki University (registration no. 17082111) and was performed in line with the Declaration of Helsinki.

Multiple immunofluorescence. Surgical specimens were fixed in $10 \%$ formaldehyde at room temperature (RT) overnight and embedded in paraffin according to a standard protocol. Serial 4- $\mu \mathrm{m}$ histological sections were deparaffinised in xylene and hydrated in descending dilutions of ethanol. All samples were stained with haematoxylin and eosin (H\&E) at RT for 4 and 2 min, respectively, and histological subtypes and pathological stage were reconfirmed. For antigen retrieval, slides were placed in a water bath at $95^{\circ} \mathrm{C}$ for $40 \mathrm{~min}$ in the citrate buffer (pH 6.0; S2031; Dako; Agilent Technologies, Inc., Santa Clara, CA, USA). Endogenous peroxidase activity was blocked by incubation with $0.3 \%$ hydrogen peroxide (catalog no. 081-04215; Wako Pure Chemical Industries, Ltd., Osaka, Japan) in 99\% methanol (catalog no. 136-01837; Fujifilm; Wako Pure Chemical Industries) for $30 \mathrm{~min}$ at RT. The slides were immersed in blocking buffer dispensed in 5\% skimmed milk (catalog no. 232100; BD Biosciences, Franklin Lakes, NJ, USA) and Triton(R) X-100 (catalog no. 35501-15; Nacalai Tesque, Inc., Kyoto, Japan) at RT for $5 \mathrm{~min}$. The blocking buffer included the following antibiotics: $0.1 \%$ streptomycin (catalog no. 85886) and $0.1 \%$ amphotericin (catalog no. A2942) (both from Sigma-Aldrich; Merck KGaA, Darmstadt, Germany). The slides were incubated at room temperature for $2 \mathrm{~h}$ with the following primary antibodies: Mouse anti-human CK14 monoclonal antibody (catalog no. ab181595; dilution, 1:100) 

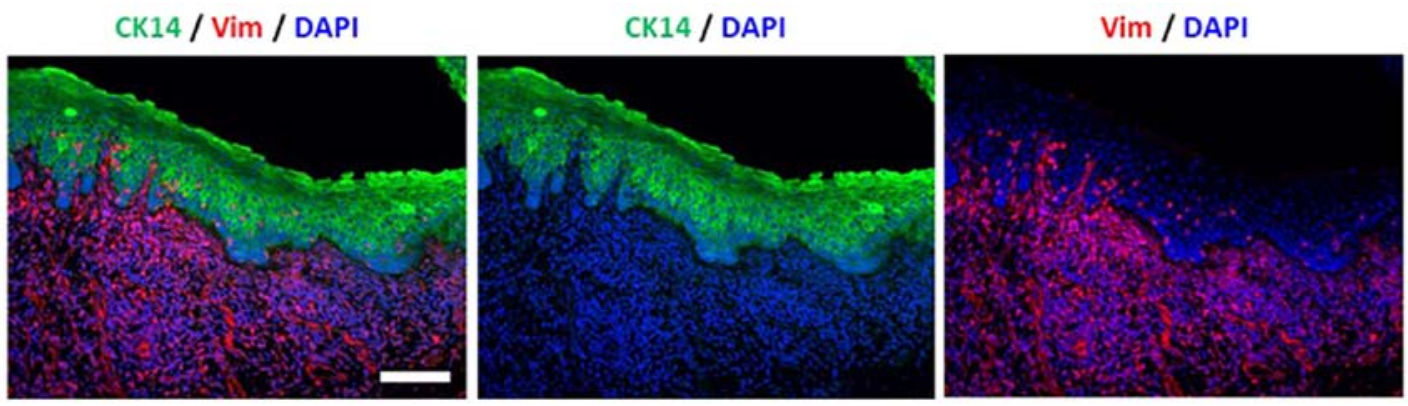

Figure 1. Specificity of anti-vimentin antibody. In the mucosal region, vimentin signals could not be detected in epithelial cells that are positive for cytokeratin 14. Scale bar, $100 \mu \mathrm{m}$. CK14, cytokeratin 14; Vim, vimentin.

to detect cancer epithelium, mouse anti-human PCNA monoclonal antibody (catalog no. ab201672; dilution, 1:100) (both from Abcam, Cambridge, UK) to evaluate the proliferative activity of the tumor; chicken anti-human vimentin monoclonal antibody (catalog no. 919101; dilution, 1:100; BioLegend, Inc., San Diego, CA, USA) and rabbit anti-human aSMA (catalog no. ab32575; dilution, 1:100; Abcam) to detect CAFs (5,7,30); and mouse anti-human E-cadherin (catalog no. 610182; dilution, 1:100; BD Biosciences) to evaluate the progression of EMT. Slides were then incubated for $1 \mathrm{~h}$ with the following secondary antibodies: Alexa Fluor 488-conjugated goat anti-mouse IgG antibody, Alexa Fluor 546-conjugated goat anti-rabbit IgG antibody or Alexa Fluor 647-conjugated goat anti-chicken antibody (Thermo Fisher Scientific, Inc., Waltham, MA, USA). Slides were washed using PBS with $10 \%$ glycerol and mounted in PBS containing DAPI. Finally, slides were sealed with a cover glass.

Images were captured using a fluorescent microscope (DM-6000B) and a digital camera (DFC-350 FX) (both from Leica Microsystems GmbH, Wetzlar, Germany) attached to the fluorescent microscope, and 3 randomly selected areas were selected to generate the optical density plots using the Histogram tool in the Image menu of Adobe Photoshop (Adobe Systems, Inc., San Jose, CA, USA). The average fluorescent intensities within the 3 randomly selected areas were recorded in arbitrary units and the relative fluorescent intensities were calculated to obtain relative levels.

Fig. 1 indicates the specificity of the anti-vimentin antibody. In the mucosal region, vimentin signals could not be detected in the epithelial cells that were positive for CK14.

Statistical analysis. All statistical analyses were conducted using SPSS for Windows Version 24.0 (IBM Corp., Armonk, NY, USA). The distributions of CAFs and expression levels of vimentin and E-cadherin in LR patients and control patients were analysed using the Mann-Whitney U test. Significance was evaluated using Fisher's exact test. $\mathrm{P}<0.05$ was considered to indicate a statistically significant difference.

\section{Results}

Patients. Based on the cancer differentiation pattern defined by the WHO, there was one case of moderately-differentiated TSCC in the LR group, while the remaining 13 patients had well-differentiated cancer. The median tumor thickness in the LR group was $5.85 \mathrm{~mm}$ (range, 4.5-11.0 mm), and was $4.5 \mathrm{~mm}$ (range, 3.0-12.0 mm) in the control group. The depth of invasion was $<10 \mathrm{~mm}$. No significant difference was detected between the two groups. Vascular, lymphatic and perineural invasion were more likely to be observed in the LR group than in the control group. In the LR group, all TSCCs recurred from the deep and/or posterior margin of the primary lesion. The mean period between surgery and the detection of LR was 8.7 months (range, 0.2-27.8 months). All LR patients underwent an additional glossectomy. However, 4 patients in the LR group (4/7 cases, 57.1\%) succumbed due to uncontrollable LR (1 cases), controllable LR (1 case), late lymph node metastasis (1 case) and lung metastases (1 case). Although one patient exhibited controllable LR, the patient made non-treatment decision. The mean follow-up period was 49.2 months for the whole series (range, 5.1-134.0 months). Table I shows a summary of all 14 patients.

Evaluation of the cancer microenvironment using multiple immunofluorescent analysis. A representative image of the LR case obtained by MIA is presented in Fig. 2. In the tumor region determined by H\&E staining (Fig. 2A and B), CK14-positive cells, which are clearly discriminated from the green autofluorescence (Fig. 2C and D), were identified as independent clusters. They were PCNA-positive (Fig. 2E and F) and were embedded in CME, which consists of vimentin-positive stromal cells (Fig. 2G and H). Comparison of the distribution between CK14-positive cancer cells (Fig. 2E and F) and vimentin-positive stromal cells indicated that stromal components appear to be present prior to the invasive propagation of cancer cells (Fig. 2G). High-magnification MIA analysis revealed that cancer clusters with high PCNA positivity were surrounded by vimentin-positive stromal cells (Fig. 2H). The authors considered spindled cells that were positive for $\alpha$ SMA and vimentin as CAFs $(5,7,30)$. CAFs were detected around the invasion front of CK14-positive cancer tissues. It should be noted that vimentin-positive stromal cells frequently express $\alpha \mathrm{SMA}$, indicating that they are CAFs (Fig $3 \mathrm{~A}$ and $\mathrm{B}$ ). Quantitative analysis of $\alpha$ SMA expression versus vimentin expression in the stromal cells revealed that activation of stromal cells was significantly accelerated in the LR group compared with the control group ( $\mathrm{P}=0.001$; Mann-Whitney U test; Fig. 3C). The present study also compared E-cadherin expression in CK14-positive cancer epithelium between the LR and control groups. As demonstrated in Fig. 4, characteristic membrane organization of E-cadherin was lost in PCNA-positive cancer clusters in the LR group. The expres- 

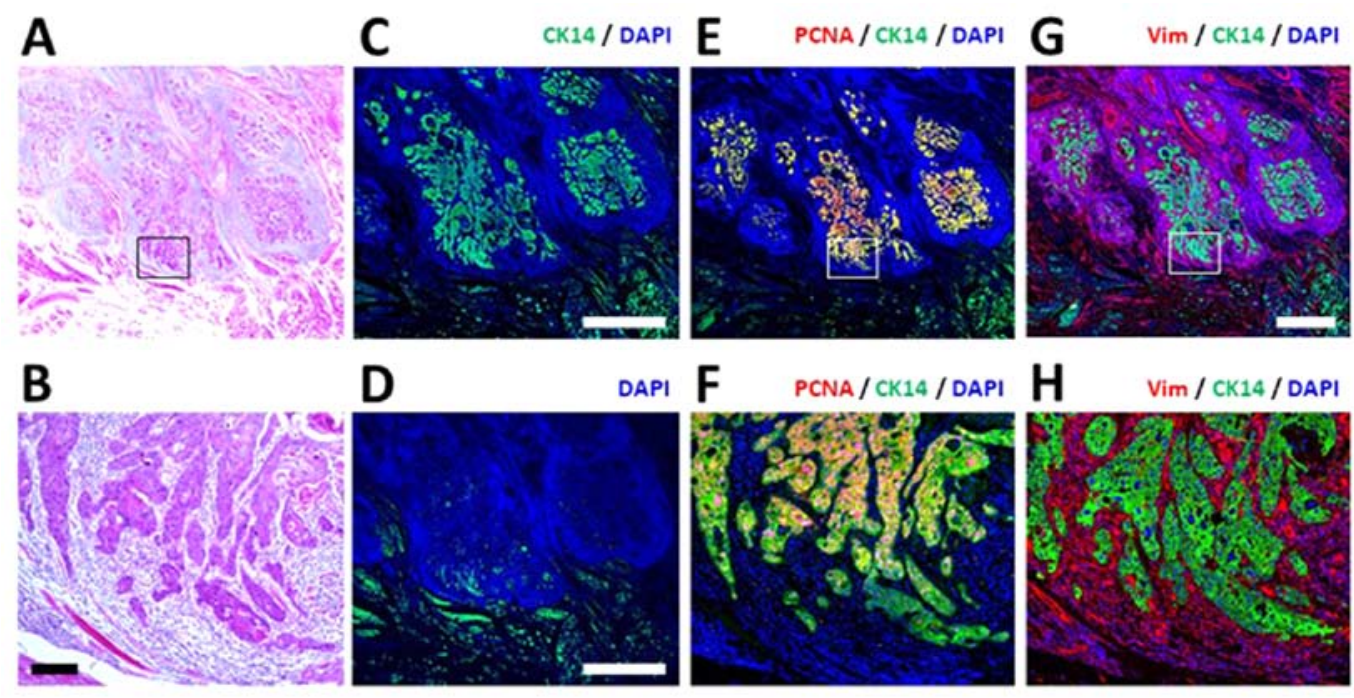

Figure 2. Multiple immunofluorescent assay of tongue squamous cell carcinoma tissue obtained from the local recurrence group. Higher magnification images of the designated area are provided in the lower panel. H\&E staining at (A) low magnification and (B) high magnification. Immunofluorescent image at (C) low magnification and (D) high magnification. CK14-positive (green) cancer cell cluster embedded in the CME consisting of CK-negative stromal cells (blue). Certain green signals outside the blue area are autofluorescence from muscle. Immunofluorescent image at (E) low magnification and (F) high magnification. A number of the invaded CK14-positive cells are also PCNA-positive. Immunofluorescent image at (G) low magnification and (H) high magnification. CK-negative CME consists of vimentin-positive stromal cells. White bar in C and G, $500 \mu \mathrm{m}$; black bar in B and white bar in D, 100 $\mu \mathrm{m}$. CK14, cytokeratin 14; Vim, vimentin; CME, cancer microenvironment; PCNA, proliferating cell nuclear antigen.
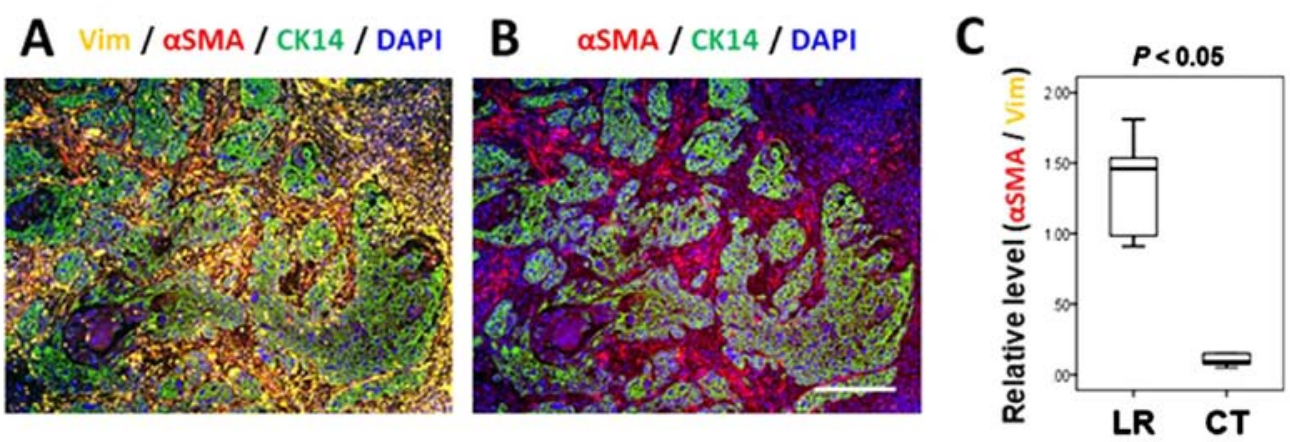

Figure 3. Multiple immunofluorescent assay in the cancer microenvironment surrounding cancer cell clusters in cases of LR. (A) CK14-positive cancer cell clusters (green) are surrounded by Vim-positive (yellow) and $\alpha$ SMA-positive (red) cancer-associated fibroblasts. (B) The expression patterns of Vim were almost matched to those of $\alpha \mathrm{SMA}$. (C) Such expression intensities of $\alpha \mathrm{SMA}$ with regards to Vim were significantly predominant in the LR cases (P=0.001). Bar, $250 \mu \mathrm{m}$. LR, local recurrence; CT, control; Vim, vimentin; CK14, cytokeratin 14; $\alpha$ SMA, $\alpha$-smooth muscle actin.

sion of E-cadherin in the CK14-positive cancer epithelia also differed significantly between the two groups $(\mathrm{P}=0.007$; Mann-Whitney U test; Fig. 4A), and the expression levels of E-cadherin, as well as the organization pattern was apparently compromised in the LR cases (Fig. 4B).

While normal CK14-positive epithelial cells did not express vimentin (Fig. 1), CK14-positive cancer cell clusters in numerous LR cases exhibited concurrent vimentin expression (Fig. 5A-E and Table II). For example, the cancer cells in the dotted area in Figure 5A, which are positive for CK14, also express vimentin. The cells exhibit epithelial morphology and CK14 expression levels equivalent to that in the normal epithelium (data not shown). Next, expression levels of vimentin within the CK14-positive cancer cells were quantified, and the Mann-Whitney $\mathrm{U}$ test revealed that the two groups were significantly different $(\mathrm{P}=0.004$; Fig. $5 \mathrm{~F})$. It should also be emphasized that such vimentin-positive cancer cells continue to exhibit normal amounts of CK14 expression, indicating that they are CK14-positive epithelial cells but that they concurrently express vimentin. The MIA also revealed various intermediate phenotypes, in which vimentin-positive cancer cells retain E-cadherin membrane distribution (Fig. 6A-C).

\section{Discussion}

The present study applied MIA to visualize the spatial distribution of CAFs in the TSCC cases with local recurrence, despite the glossectomy with adequate safety margins having been performed. The results of the present study clearly demonstrated that the distribution of CAFs was significantly associated with LR. While the distribution of vimentin-positive stromal cells was equivalent between the LR and control cases, vimentinpositive cells frequently co-expressed $\alpha$ SMA in the LR cases.

It has recently been reported that CAFs occurring in high frequency in oral SCCs are associated with cancer recurrence $(8,31)$. Furthermore, CAFs are key factors in the 
A

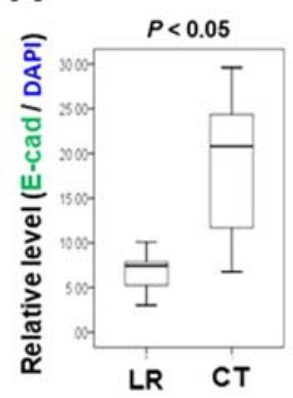

B

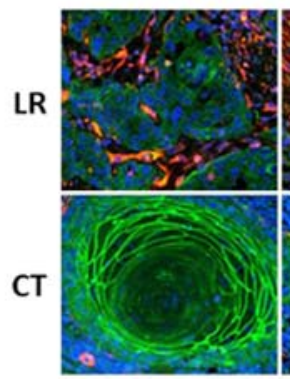

E-cad / Vim / DAPI
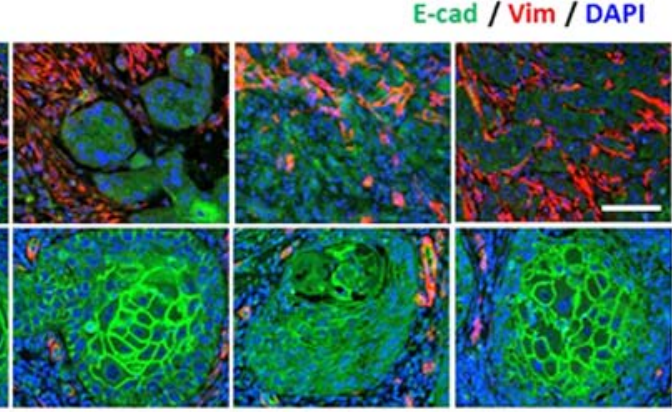

Figure 4. Expression patterns of E-cad. (A) Expression levels of E-cad are significantly higher in the CT cases. The Mann-Whitney U test revealed a significant difference in expression levels ( $\mathrm{P}=0.007$ ). (B) Comparison of the membrane pattern of E-cad between the LR and CT cases. Scale bars, $50 \mu \mathrm{m}$. Bar, $50 \mu \mathrm{m}$. $\mathrm{LR}$, local recurrence; CT, control; E-cad, E-cadherin; Vim, vimentin.
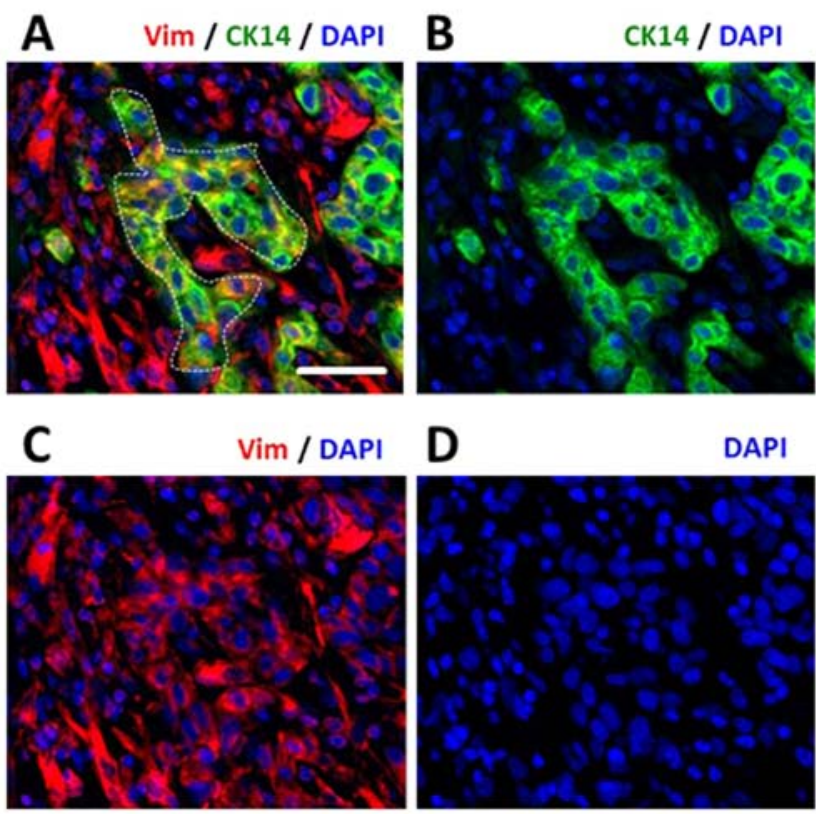

D

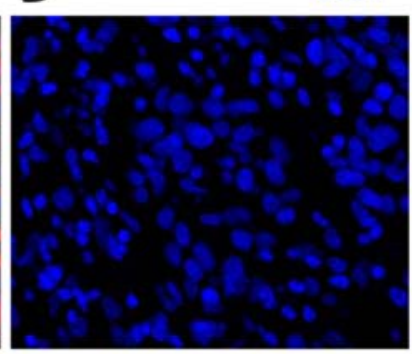

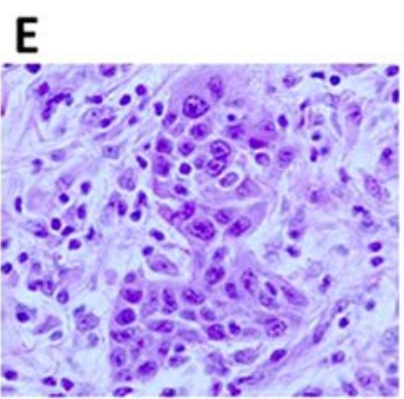

F

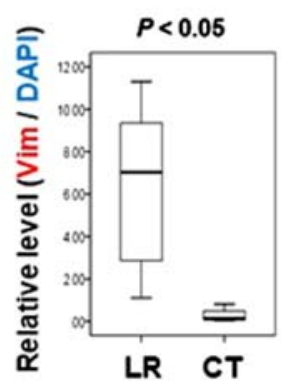

Figure 5. Vim expression in CK14-positive cancer cells. (A) Multiple immunofluorescent assay revealed co-expression of Vim in CK14-positive cancer cells, which are marked by a dotted line. (B) The CK14-positive area also expresses (C) vimentin, and the fluorescence intensities in a dotted area were quantified. (D) DAPI staining. (E) H\&E staining image of the same site in the same case. (F) Vim expression in CK14-positive cancer cells is exclusively observed in the LR cases. A Mann-Whitney U test revealed that the two groups differed significantly (P=0.004). Bar, 50 $\mu \mathrm{m}$. Vim, vimentin; CK14, cytokeratin 14; LR, local recurrence; CT, control.

collective invasion of cancer cells as they are capable of remodelling the extracellular matrix and providing the mechanical propulsive forces required to facilitate tumor invasion (8). Epithelial cancer cells are able to activate CAFs, an interaction that is mediated by ligands, including tumor necrosis factor- $\alpha$ (TNF- $\alpha$ ) and interleukin- $\alpha / \beta$ (IL- $1 \alpha / \beta)$. CAFs secrete molecules, including TNF- $\alpha$, IL- $1 \alpha / \beta$, IL-33, CCL7, SDF-1, MDNF, type 1 collagen, HGF, IGF2, BMP4, MMPs, PGE2, KGF, activin A and PDGF, and microRNAs (32). In this way, pre-cancerous cell growth (33), cancer cell migration and invasion, metastasis, angiogenesis, immune system evasion (34) and chemotherapy resistance (35) are promoted. Additionally, it has been reported that a CAF-rich reactive stroma is associated with high-grade malignancies and a poor prognosis in oral cancer (36). Therefore, these reports support the results of the present study, in which activation of stromal cells are associated with local recurrence.
In the present study, 6/7 patients in the LR group exhibited perineural and/or vascular invasion and a poor prognosis. Certain studies have reported that perineural and vascular invasion are prognostic factors for regional metastasis and poor outcomes in patients with TSCC, although they are not significant associated with local recurrence (37).

To date, a diagnosis of EMT has been given when epithelial cancer cells express vimentin and lose E-cadherin. However, the expression of epithelial markers, including CKs, in epithelial cancer cells was not taken into consideration, since general tissue staining methods do not permit the examination of simultaneous expression of multiple markers in the same cell. Therefore, MIA was introduced and it was demonstrated that vimentin-positive cancer cells retained CK14 expression. CK14 expression was confirmed to be equivalent to that observed in the normal epithelial cells. It is therefore not adequate to utilize EMT or partial EMT to describe the 
Table I. Patient characteristics and outcomes.

\begin{tabular}{|c|c|c|c|c|c|c|c|}
\hline & Sex & Age & TNM & $\begin{array}{l}\text { Clinical growth } \\
\text { pattern pattern }\end{array}$ & Differentiation & $\begin{array}{l}\text { Vascular, lymphatic } \\
\text { and perineural invasion }\end{array}$ & Outcome \\
\hline \multicolumn{8}{|c|}{ LR group } \\
\hline Case 1 & M & 39 & T2N0M0 & Invasive & Well & - & DFS \\
\hline Case 2 & M & 75 & T1N0M0 & Invasive & Moderate & $\mathrm{v}$ & $\mathrm{N}$ mortality \\
\hline Case 3 & M & 57 & T2N0M0 & Invasive & Well & $\mathrm{v}$ & Other death \\
\hline Case 4 & $\mathrm{~F}$ & 62 & T2N0MO & Invasive & Well & ly, v, pn & DFS \\
\hline Case 5 & $\mathrm{M}$ & 79 & T2N0M0 & Invasive & Well & $\mathrm{v}, \mathrm{pn}$ & T mortality \\
\hline Case 6 & $\mathrm{M}$ & 72 & $\mathrm{~T} 2 \mathrm{~N} 1 \mathrm{M} 0$ & Unknown & Well & ly, v, pn & T mortality \\
\hline Case 7 & $\mathrm{~F}$ & 46 & T2N0M0 & Expansive & Well & pn & DFS \\
\hline \multicolumn{8}{|c|}{ Control group } \\
\hline Case 1 & M & 63 & T2N0M0 & Superficial & Well & $\mathrm{v}$ & DFS \\
\hline Case 2 & M & 52 & T2N0M0 & Invasive & Well & - & DFS \\
\hline Case 3 & M & 61 & T1N0M0 & Superficial & Well & - & DFS \\
\hline Case 4 & $\mathrm{~F}$ & 64 & T2N0M0 & Invasive & Well & $\mathrm{v}$ & DFS \\
\hline Case 5 & $\mathrm{~F}$ & 61 & T2N0M0 & Superficial & well & $\mathrm{v}, \mathrm{pn}$ & DFS \\
\hline Case 6 & M & 62 & T2N0M0 & Invasive & well & ly, v & DFS \\
\hline Case 7 & M & 86 & T2N0M0 & Invasive & well & $\mathrm{v}$ & DFS \\
\hline
\end{tabular}

TNM, Tumor-Node-Metastasis; M, male; F, female; LR, local recurrence; lv, lymphatic vessel invasion; v, vascular invasion; pn, perineural invasion; DFS, disease-free survival.

Table II. Relative expression of aSMA, Vimentin and E-cadherin.

\begin{tabular}{lccc}
\hline & $\begin{array}{c}\alpha \text { SMA } \\
(\alpha \text { SMA } / \\
\text { Vimentin })\end{array}$ & $\begin{array}{c}\text { Vimentin } \\
\text { (Vimentin/ } \\
\text { DAPI })\end{array}$ & $\begin{array}{c}\text { E-cadherin } \\
(\text { E-cadherin/ } \\
\text { DAPI) }\end{array}$ \\
\hline LR group & & & \\
Case 1 & $0.94 \pm 0.12$ & $2.93 \pm 0.74$ & $7.46 \pm 0.99$ \\
Case 2 & $1.56 \pm 0.42$ & $2.80 \pm 0.68$ & $8.15 \pm 1.52$ \\
Case 3 & $1.46 \pm 0.21$ & $8.31 \pm 1.20$ & $3.0 \pm 1.34$ \\
Case 4 & $1.81 \pm 0.27$ & $7.03 \pm 1.43$ & $5.83 \pm 1.30$ \\
Case 5 & $1.51 \pm 0.44$ & $11.3 \pm 2.86$ & $4.67 \pm 1.90$ \\
Case 6 & $1.01 \pm 0.18$ & $10.4 \pm 1.83$ & $7.60 \pm 1.16$ \\
Case 7 & $0.93 \pm 0.20$ & $5.59 \pm 1.06$ & $6.75 \pm 1.72$ \\
Control group & & & \\
Case 1 & $0.06 \pm 0.01$ & $0.14 \pm 0.07$ & $29.6 \pm 3.46$ \\
Case 2 & $0.09 \pm 0.03$ & $0.13 \pm 0.08$ & $25.3 \pm 3.12$ \\
Case 3 & $0.15 \pm 0.07$ & $0.02 \pm 0.01$ & $12.9 \pm 1.32$ \\
Case 4 & $0.09 \pm 0.06$ & $0.15 \pm 0.09$ & $20.8 \pm 2.86$ \\
Case 5 & $0.91 \pm 0.19$ & $1.11 \pm 0.51$ & $10.1 \pm 2.11$ \\
Case 6 & $0.05 \pm 0.03$ & $0.82 \pm 0.29$ & $10.5 \pm 2.10$ \\
Case 7 & $0.15 \pm 0.06$ & $0.02 \pm 0.01$ & $23.4 \pm 6.31$ \\
\hline
\end{tabular}

$\alpha$ SMA, $\alpha$-smooth muscle actin; LR, local recurrence.

results of the present study. Thus, the concept of APT was proposed, in which epithelial cancer cells dedifferentiate into more primitive states (Fig. 7). During embryonic develop- ment, it is well described that vimentin is co-expressed with $\mathrm{CKs}$ in the mesoderm, since the mesoderm originates from vimentin-positive epiblasts. Next, specification of epithelial cells limit the expression of vimentin, and therefore, epithelial cells do not express any vimentin protein. The present study observed that CK14-positive cells co-express vimentin, indicating that epithelial cells obtained more primitive features, including epiblasts, which should be separated from concepts of EMT and partial EMT. Notably, it has been reported that vimentin co-exists with CK14 in the migrating epithelial cells (38). There are also studies demonstrating that vimentin and CKs are co-expressed in epithelial cancer cells $(27,39,40)$. This result was also observed in the LR group; therefore, we hypothesized that APT may be a prognostic marker of LR and aid in improving the understanding of LR in patients with early-stage TSCC. Clinically, patients with APT should undergo frequent examinations using contrast enhanced-CT, ultrasound and positron-emission tomography if required, in addition to inspection during postoperative follow-up; if clinician can perform an early diagnosis, they will be able to begin treatment of the LR tumor sooner.

It was also noted that one major limitation of the present study was the small number of early-staged TSCC patients with LR ( $=7)$, which may have affected the statistical results. To begin with, the number of these cases was relatively small. Since almost all cases $(98.5 \%)$ postoperatively elapsed without LR, further accumulation of LR cases to hone accuracy of statistics is required in the future.

MIA also enabled the detection of pleiotropic phenotypes of cancer cells. For example, as demonstrated in Figure 6, vimentin and E-cadherin are co-expressed in the same cells, indicating that EMT is not an all-or-none phenomena. Such 


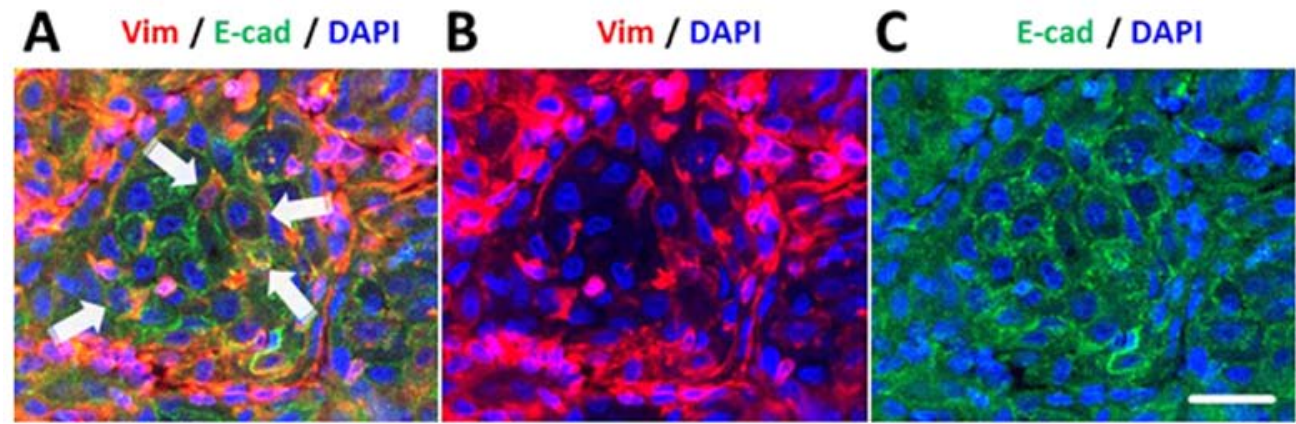

Figure 6. Representative image of concurrent expression of Vim and E-cad in the LR group. (A) Merged image demonstrates the membrane organization of E-cadherin in Vim-positive cells (indicated by white arrows). (B) Vimentin-positive and (C) E-cadherin-positive cancer cells. Bar, $25 \mu \mathrm{m}$.

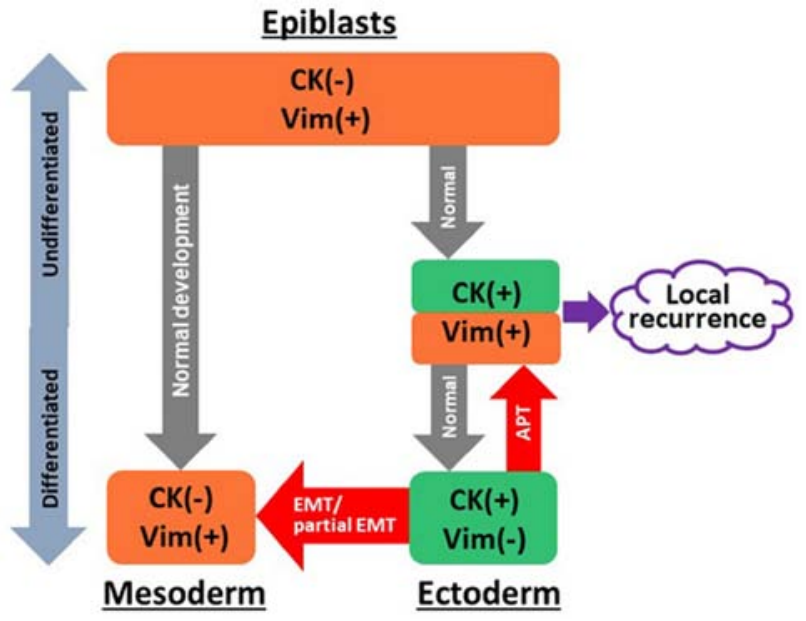

Figure 7. A concept map of APT. In the normal process of ectoderm development and differentiation, vimentin-positive epiblasts are differentiated to ectoderm with upregulation of CKs and downregulation of Vim. EMT or partial EMT is designated as just a transition from ectoderm to mesoderm. While ectoderm specification limits Vim expression in epithelial cells, local recurrence cases exhibit APT, in which CK14-positive cells co-express vimentin. The APT concept is totally different from EMT, which is the direct conversion of epithelial cells to mesenchymal cells that only express vimentin. APT, anaplastic transition; EMT, epithelial-mesenchymal transition; CK, cytokeratin; Vim, vimentin.

intermediate phenotypes have been referred to as partial EMT. Grigore et al (41) discussed the concept of partial EMT in associated with tumor budding at the invasion front, which was associated with decreased E-cadherin and increased vimentin levels, suggesting that it may be a morphological feature of EMT. Wang et al (27) performed immunohistochemical analysis with pan-CK staining and revealed that budding cells scattered in the stroma were also positive for vimentin. The results of the present study not only confirmed those of previous reports, but also provided the concept of APT, which may aid in dissolving controversial reports on partial EMT.

In current clinical practice, clinicians tend to diagnose EMT when epithelial cancer cells loose E-cadherin and express vimentin. However, the diagnosis does not include an evaluation of CK expression, the specific nature of epithelia. A more detailed diagnosis of the degree of malignancy includes the evaluation of the histopathological features with HE staining, as well as MIA using CK and vimentin. The analysis of expression of transcriptional factors, including Snail, Slug, ZEB1,
Twist and Bmil, has also been used to evaluate EMT progression and the degree of malignancy (42), and the analysis of changes in expression of these genes will also provide us with a more detailed mechanism through which APT is achieved.

In conclusion, a novel concept of APT was proposed, in which epithelial cancer cells concurrently obtain mesenchymal features. MIA first enabled us to evaluate the residual expression of CK in epithelial cancer cells that have lost E-cadherin and begun to express vimentin. Since APT is significantly associated with the LR, it can be used as a predictive marker for LR in patients with early-stage TSCC.

\section{Acknowledgements}

Not applicable.

\section{Funding}

No funding was received.

\section{Availability of data and materials}

The datasets used and/or analyzed during the current study are available from the corresponding author on reasonable request.

\section{Author contributions}

KO and KS contributed toward conception and design of the study, data acquisition, analysis and interpretation, and drafting of the manuscript. KO and HT prepared sample slides. KO, SY, TN and MU contributed toward data analysis. KS, MU and SY critically revised the manuscript. All authors approved the final manuscript and agree to be accountable for all aspects of the work.

\section{Ethics approval and consent to participate}

The present study was performed according to the Declaration of Helsinki on medical protocol and ethics and was approved by the regional Ethical Review Board of Nagasaki University (Nagasaki, Japan; registration no. 17082111).

\section{Patient consent for publication}

Not applicable. 


\section{Competing interests}

The authors declare that they have no competing interests.

\section{References}

1. Lin NN, Wang P, Zhao D, Zhang FJ, Yang K and Chen R: Significance of oral cancer-associated fibroblasts in angiogenesis lymphangiogenesis, and tumor invasion in oral squamous cell carcinoma. J Oral Pathol Med 46: 21-30, 2017.

2. Haddad RI and Shin DM: Recent advances in head and neck cancer. N Engl J Med 359: 1143-1154, 2008.

3. Döbrossy L: Epidemiology of head and neck cancer: Magnitude of the problem. Cancer Metastasis Rev 24: 9-17, 2005.

4. Rodrigues-Lisoni FC, Peitl P Jr, Vidotto A, Polachini GM, Maniglia JV, Carmona-Raphe J, Cunha BR, Henrique T, Souza CF, Teixeira RA, et al; Head and Neck Genome Project GENCAPO: Genomics and proteomics approaches to the study of cancer-stroma interactions. BMC Med Genomics 3: 14, 2010.

5. Kalluri R and Zeisberg M: Fibroblasts in cancer. Nat Rev Cancer 6: 392-401, 2006.

6. Hanna E, Quick J and Libutti SK: The tumour microenvironment A novel target for cancer therapy. Oral Dis 15: 8-17, 2009.

7. Zhou B, Chen WL, Wang YY, Lin ZY, Zhang DM, Fan S and Li JS: A role for cancer-associated fibroblasts in inducing the epithelial-to-mesenchymal transition in human tongue squamous cell carcinoma. J Oral Pathol Med 43: 585-592, 2014.

8. Vered M, Dayan D, Yahalom R, Dobriyan A, Barshack I, Bello IO, Kantola S and Salo T: Cancer-associated fibroblasts and epithelial-mesenchymal transition in metastatic oral tongue squamous cell carcinoma. Int J Cancer 127: 1356-1362, 2010.

9. Li H, Zhang J, Chen SW, Liu LL, Li L, Gao F, Zhuang SM, Wang LP, Li Y and Song M: Cancer-associated fibroblasts provide a suitable microenvironment for tumor development and progression in oral tongue squamous cancer. J Transl Med 13: $198,2015$.

10. De Wever O and Mareel M: Role of tissue stroma in cancer cell invasion. J Pathol 200: 429-447, 2003.

11. Jewett A, Head C and Cacalano NA: Emerging mechanisms of immunosuppression in oral cancers. J Dent Res 85: 1061-1073, 2006.

12. De Wever O, Demetter P, Mareel M and Bracke M: Stromal myofibroblasts are drivers of invasive cancer growth. Int J Cancer 123: 2229-2238, 2008.

13. Wheeler SE, Shi H, Lin F, Dasari S, Bednash J, Thorne S, Watkins S, Joshi R and Thomas SM: Enhancement of head and neck squamous cell carcinoma proliferation, invasion, and metastasis by tumor-associated fibroblasts in preclinical models. Head Neck 36: 385-392, 2014.

14. Sappino AP, Skalli O, Jackson B, Schürch W and Gabbiani G: Smooth-muscle differentiation in stromal cells of malignant and non-malignant breast tissues. Int J Cancer 41: 707-712, 1988.

15. Lazard D, Sastre X, Frid MG, Glukhova MA, Thiery JP and Koteliansky VE: Expression of smooth muscle-specific proteins in myoepithelium and stromal myofibroblasts of normal and malignant human breast tissue. Proc Natl Acad Sci USA 90: 999-1003, 1993

16. Mueller MM and Fusenig NE: Friends or foes - bipolar effects of the tumour stroma in cancer. Nat Rev Cancer 4: 839-849, 2004.

17. Rønnov-Jessen L, Petersen OW and Bissell MJ: Cellular changes involved in conversion of normal to malignant breast: Importance of the stromal reaction. Physiol Rev 76: 69-125, 1996.

18. el-Naggar AK, Lai S, Luna MA, Zhou XD, Weber RS, Goepfert H and Batsakis JG: Sequential p53 mutation analysis of pre-invasive and invasive head and neck squamous carcinoma. Int J Cancer 64: 196-201, 1995.

19. Kellermann MG, Sobral LM, da Silva SD, Zecchin KG, Graner E, Lopes MA, Kowalski LP and Coletta RD: Mutual paracrine effects of oral squamous cell carcinoma cells and normal oral fibroblasts: Induction of fibroblast to myofibroblast transdifferentiation and modulation of tumor cell proliferation. Oral Oncol 44: 509-517, 2008.

20. Daly AJ, McIlreavey L and Irwin CR: Regulation of HGF and SDF-1 expression by oral fibroblasts--implications for invasion of oral cancer. Oral Oncol 44: 646-651, 2008.

21. Thiery JP, Acloque H, Huang RY and Nieto MA: Epithelial-mesenchymal transitions in development and disease. Cell 139: 871-890, 2009.
22. PotentaS,ZeisbergEand KalluriR:Therole of endothelial-to-mesenchymal transition in cancer progression. Br J Cancer 99: 1375-1379, 2008.

23. Kalluri R and Weinberg RA: The basics of epithelial-mesenchymal transition. J Clin Invest 119: 1420-1428, 2009.

24. Chen C, Zimmermann M, Tinhofer I, Kaufmann AM and Albers AE: Epithelial-to-mesenchymal transition and cancer stem(-like) cells in head and neck squamous cell carcinoma. Cancer Lett 338: 47-56, 2013.

25. Lee JM, Dedhar S, Kalluri R and Thompson EW: The epithelialmesenchymal transition: New insights in signaling, development, and disease. J Cell Biol 172: 973-981, 2006.

26. Yang J, Mani SA, Donaher JL, Ramaswamy S, Itzykson RA, Come C, Savagner P, Gitelman I, Richardson A and Weinberg RA: Twist, a master regulator of morphogenesis, plays an essential role in tumor metastasis. Cell 117: 927-939, 2004.

27. Wang C, Huang H, Huang Z, Wang A, Chen X, Huang L, Zhou X and Liu X: Tumor budding correlates with poor prognosis and epithelial-mesenchymal transition in tongue squamous cell carcinoma. J Oral Pathol Med 40: 545-551, 2011.

28. Lydiatt WM, Patel SG, O'Sullivan B, Brandwein MS, Ridge JA, Migliacci JC, Loomis AM and Shah JP: Head and neck cancersmajor changes in the American Joint Committee on cancer eighth edition cancer staging manual. CA Cancer J Clin 67: 122-37, 2017.

29. Brierley JD, Gospodarowicz MK and Wittekind C: TNM Classification of Malignant Tumours. 8th edition. Wiley-Blackwell, Chichester, 2017.

30. Lim KP, Cirillo N, Hassona Y, Wei W, Thurlow JK, Cheong SC, Pitiyage G, Parkinson EK and Prime SS: Fibroblast gene expression profile reflects the stage of tumour progression in oral squamous cell carcinoma. J Pathol 223: 459-469, 2011.

31. Kawashiri S, Tanaka A, Noguchi N, Hase T, Nakaya H, Ohara T, Kato K and Yamamoto E: Significance of stromal desmoplasia and myofibroblast appearance at the invasive front in squamous cell carcinoma of the oral cavity. Head Neck 31: 1346-1353, 2009.

32. Wang J, Min A, Gao S and Tang Z: Genetic regulation and potentially therapeutic application of cancer-associated fibroblasts in oral cancer. J Oral Pathol Med 43: 323-334, 2014.

33. Olumi AF, Grossfeld GD, Hayward SW, Carroll PR, Tlsty TD and Cunha GR: Carcinoma-associated fibroblasts direct tumor progression of initiated human prostatic epithelium. Cancer Res 59: 5002-5011, 1999.

34. Takahashi H, Sakakura K, Kudo T, Toyoda M, Kaira K, Oyama T and Chikamatsu K: Cancer-associated fibroblasts promote an immunosuppressive microenvironment through the induction and accumulation of protumoral macrophages. Oncotarget 8: 8633-8647, 2017.

35. Johansson AC, Ansell A, Jerhammar F, Lindh MB, Grénman R, Munck-Wikland E, Östman A and Roberg K: Cancer-associated fibroblasts induce matrix metalloproteinase-mediated cetuximab resistance in head and neck squamous cell carcinoma cells. Mol Cancer Res 10: 1158-1168, 2012.

36. Sinha N, Mukhopadhyay S, Das DN, Panda PK and Bhutia SK: Relevance of cancer initiating/stem cells in carcinogenesis and therapy resistance in oral cancer. Oral Oncol 49: 854-862, 2013.

37. Matsushita Y, Yanamoto S, Takahashi H, Yamada S, Naruse T, Sakamoto Y, Ikeda H, Shiraishi T, Fujita S, Ikeda T, et al: A clinicopathological study of perineural invasion and vascular invasion in oral tongue squamous cell carcinoma. Int J Oral Maxillofac Surg 44: 543-548, 2015.

38. Velez-delValle C, Marsch-Moreno M, Castro-Muñozledo F, Galván-Mendoza IJ and Kuri-Harcuch W: Epithelial cell migration requires the interaction between the vimentin and keratin intermediate filaments. Sci Rep 6: 24389, 2016.

39. Ramaekers FC, Haag D, Kant A, Moesker O, Jap PH and Vooijs GP: Coexpression of keratin- and vimentin-type intermediate filaments in human metastatic carcinoma cells. Proc Natl Acad Sci USA 80: 2618-2622, 1983.

40. Pagan R, Martín I, Alonso A, Llobera M and Vilaró S: Vimentin filaments follow the preexisting cytokeratin network during epithelial-mesenchymal transition of cultured neonatal rat hepatocytes. Exp Cell Res 222: 333-344, 1996.

41. Grigore AD, Jolly MK, Jia D, Farach-Carson MC and Levine H: Tumor Budding: The Name is EMT. Partial EMT. J Clin Med 5: E51, 2016.

42. Pradella D, Naro C, Sette C and Ghigna C: EMT and stemness: Flexible processes tuned by alternative splicing in development and cancer progression. Mol Cancer 16: 8, 2017. 